\title{
Correction to: Spin-label Order Parameter Calibrations for Slow Motion
}

\section{Derek Marsh $^{1,2}$}

Published online: 6 July 2019

(c) Springer-Verlag GmbH Austria, part of Springer Nature 2019

\section{Correction to: Appl Magn Reson (2018) 49:97-106 https://doi.org/10.1007/s00723-017-0940-7}

In the print published article, some equations were published incorrectly, the correct equations are given below.

Equation 7 of the above manuscript contains an error of sign and should read:

$$
S_{z z}=\frac{\left(A_{\max }-A_{\min }\right)-\left(d_{\max }^{o}+d_{\min }^{o}\right)}{\Delta A-\left(d_{\max }^{\prime}+d_{\min }^{\prime}\right)} .
$$

Because of this, calibration parameters for deducing order parameters $S_{z z}$ (and isotropic hyperfine couplings $a_{o}$ ) from the measurables $A_{\max }$ and $A_{\min }$, which appear in Tables 1 and 2 are incorrect. The correct calibration parameters are listed in the new Tables 1 and 2 given here. The calibration equations to which these tables refer are:

$$
S_{z z}=s_{1} \times\left(A_{\max }-A_{\min }\right)-s_{o}
$$

and

$$
a_{o}=\frac{1}{3}\left(f_{\max } A_{\max }+f_{\min } A_{\min }\right)+\delta a_{o},
$$

respectively.

With the exception of equations that incorporate numerical constants, the paper otherwise remains unchanged. Corrected versions of the latter are:

$$
s_{1}=40.4-5.21 \times \log _{10} D_{R \perp}\left(\mathrm{s}^{-1}\right)
$$

The original article can be found online at https://doi.org/10.1007/s00723-017-0940-7.

Derek Marsh

dmarsh@gwdg.de

1 Max-Planck-Institut für biophysikalische Chemie, 37070 Göttingen, Germany

2 MEMPHYS-Centre for Biomembrane Physics, University of Southern Denmark, Campusvej 55, 5230 Odense M, Denmark 
Table 1 Calibration parameters in Eqs. 8 and 13 for order parameter and isotropic hyperfine coupling, for different diffusion coefficients $D_{R \perp}\left(=0.1 \times D_{R / /}\right)$

\begin{tabular}{llllll}
\hline$D_{R \perp}\left(\mathrm{s}^{-1}\right)$ & $s_{1}\left(\mathrm{mT}^{-1}\right)$ & $s_{o}$ & $f_{\max }$ & $f_{\min }$ & $\delta a_{o}(\mathrm{mT})$ \\
\hline $1.5 \times 10^{7}$ & 2.25805 & 4.55239 & 1.08401 & 1.91599 & -0.17575 \\
$2.0 \times 10^{7}$ & 2.35755 & 4.62642 & 1.10419 & 1.89581 & -0.21582 \\
$2.4 \times 10^{7}$ & 1.9727 & 3.67669 & 1.07009 & 1.92991 & -0.13875 \\
$2.65 \times 10^{7}$ & 1.69563 & 3.02791 & 1.051 & 1.949 & -0.09576 \\
$3.0 \times 10^{7}$ & 1.33364 & 2.20281 & 1.01063 & 1.98937 & -0.00925 \\
$4.0 \times 10^{7}$ & 0.82337 & 1.04946 & 0.97798 & 2.02202 & 0.0629 \\
$5.0 \times 10^{7}$ & 0.6322 & 0.6186 & 0.97215 & 2.02785 & 0.07795 \\
$6.0 \times 10^{7}$ & 0.55514 & 0.43971 & 0.97219 & 2.02781 & 0.0793 \\
$7.0 \times 10^{7}$ & 0.48987 & 0.29547 & 0.96391 & 2.03609 & 0.09822 \\
$8.0 \times 10^{7}$ & 0.47378 & 0.25449 & 0.97708 & 2.02292 & 0.07194 \\
$1.0 \times 10^{8}$ & 0.43282 & 0.16264 & 0.97892 & 2.02108 & 0.06986 \\
$1.2 \times 10^{8}$ & 0.41242 & 0.11679 & 0.98246 & 2.01754 & 0.06369 \\
$1.5 \times 10^{8}$ & 0.39803 & 0.08341 & 0.98445 & 2.01555 & 0.05915 \\
& $(0.3824$ & 0.04808 & 0.97197 & 2.02803 & $0.08735)^{\mathrm{a}}$ \\
$2.0 \times 10^{8}$ & 0.38495 & 0.05415 & 0.98742 & 2.01258 & 0.05318 \\
& $(0.37738$ & 0.0371 & 0.9811 & 2.0189 & $0.06739)^{\mathrm{a}}$ \\
\hline
\end{tabular}

Empirical logistic fits: $s_{1}\left(\mathrm{mT}^{-1}\right)=\frac{1.980}{1+\left(D_{R} / 2.979 \times 10^{7}\right)^{5.23}}+0.444$

$s_{0}=\frac{4.750}{1+\left(D_{R} / 2.908 \times 10^{7}\right)^{5.028}}+0.185$

$f_{\text {max }}=0.977+\frac{0.116}{1+\left(D_{R} / 2.77 \times 10^{7}\right)^{11.67}}$

$f_{\text {min }}=2.023-\frac{0.116}{1+\left(D_{R} / 2.77 \times 10^{7}\right)^{11.67}}$

$\delta a_{o}=0.071-\frac{0.267}{1+\left(D_{R} / 2.77 \times 10^{7}\right)^{10.84}}$

${ }^{\mathrm{a}}$ Fits with $d_{\text {max }}^{o}=0, d_{\max }^{\prime}=0$
Table 2 Calibration parameters in Eqs. 8 and 13 for order parameter and isotropic hyperfine coupling, for different diffusion coefficients $D_{R} \equiv D_{R \perp}\left(=D_{R / /}\right)$

\begin{tabular}{lcllll}
\hline$D_{R}\left(\mathrm{~s}^{-1}\right)$ & $s_{1}\left(\mathrm{mT}^{-1}\right)$ & $s_{o}$ & $f_{\max }$ & $f_{\min }$ & $\delta a_{o}(\mathrm{mT})$ \\
\hline $1.5 \times 10^{7}$ & 2.01438 & 3.99366 & 1.14364 & 1.85636 & -0.31248 \\
$2.0 \times 10^{7}$ & 2.24173 & 4.36568 & 1.12865 & 1.87135 & -0.2714 \\
$2.4 \times 10^{7}$ & 1.89154 & 3.49836 & 1.09147 & 1.90853 & -0.18617 \\
$2.65 \times 10^{7}$ & 1.6547 & 2.93689 & 1.06587 & 1.93413 & -0.12903 \\
$3.0 \times 10^{7}$ & 1.33214 & 2.19694 & 1.02646 & 1.97354 & -0.04372 \\
$4.0 \times 10^{7}$ & 0.80587 & 1.01428 & 0.99621 & 2.00379 & 0.02409 \\
$5.0 \times 10^{7}$ & 0.66375 & 0.68161 & 0.99214 & 2.00786 & 0.03678 \\
$6.0 \times 10^{7}$ & 0.56446 & 0.45829 & 0.98075 & 2.01925 & 0.06204 \\
$7.0 \times 10^{7}$ & 0.48962 & 0.29509 & 0.96673 & 2.03327 & 0.0917 \\
$8.0 \times 10^{7}$ & 0.45 & 0.20786 & 0.96319 & 2.03681 & 0.09983 \\
$1.0 \times 10^{8}$ & 0.43535 & 0.16771 & 0.98012 & 2.01988 & 0.06687 \\
$1.2 \times 10^{8}$ & 0.41288 & 0.11771 & 0.98171 & 2.01829 & 0.06421 \\
$1.5 \times 10^{8}$ & 0.39729 & 0.08246 & 0.98571 & 2.01429 & 0.05752 \\
& $(0.38172$ & 0.04724 & 0.9732 & 2.0268 & $0.08581)^{\mathrm{a}}$ \\
$2.0 \times 10^{8}$ & 0.38495 & 0.05415 & 0.98742 & 2.01258 & 0.05318 \\
& $(0.37738$ & 0.0371 & 0.9811 & 2.0189 & $0.06739)^{\mathrm{a}}$ \\
\hline $\mathrm{Fits} w$ & & & & &
\end{tabular}

${ }^{\mathrm{a}}$ Fits with $d_{\max }^{o}=0, d_{\max }^{\prime}=0$ 


$$
s_{o}=92.9-12.10 \times \log _{10} D_{R \perp}\left(\mathrm{s}^{-1}\right)
$$

over the range $D_{R \perp}=(2.0-4.0) \times 10^{7} \mathrm{~s}^{-1}$.

$$
\begin{gathered}
S_{z z}=0.380 \times\left(A_{\max }-A_{\min }\right)-0.043 \\
f_{\max }=4.27-0.433 \times \log _{10} D_{R \perp}\left(\mathrm{s}^{-1}\right) \\
f_{\min }=-1.27+0.433 \times \log _{10} D_{R \perp}\left(\mathrm{s}^{-1}\right) \\
\delta a_{o}(\mathrm{mT})=-7.18+0.955 \times \log _{10} D_{R \perp}\left(\mathrm{s}^{-1}\right),
\end{gathered}
$$

over the range $D_{R \perp}=(2.0-4.0) \times 10^{7} \mathrm{~s}^{-1}$.

$$
a_{o}(\mathrm{mT})=\frac{1}{3}\left(0.976 A_{\max }+2.023 A_{\min }\right)+0.077 .
$$

I am most grateful to Prof. Ana Gennaro for alerting me to this problem; and I apologise to workers in the field for the inconvenience caused.

Publisher's Note Springer Nature remains neutral with regard to jurisdictional claims in published maps and institutional affiliations. 